\title{
Le sondeur infrarouge IASI
}

Elsa JACQUETTE, Elisa BALDIT ef l'équipe projet IASI CNES

Quel temps fera-t-il demain? Quelle est la qualité de l'air ? Quels sont les signes du réchauffement de notre planète ? Les avions pourront-ils voler suite à l'éruption volcanique de l'Eyjafjallajökull ? Les enjeux de la météorologie, de la chimie atmosphérique et de la climatologie sont nombreux et de plus en plus présents dans notre société et notre quotidien. IASI, Interféromètre Atmosphérique de Sondage Infrarouge, a été développé pour fournir des données pour la communauté météorologique opérationnelle et les communautés scientifiques en chimie atmosphérique et en climatologie afin de répondre à toutes ces questions.

\begin{abstract}
ASl est un spectro-radiomètre embarqué sur les satellites météorologiques européens en orbite polaire MetOp, développé par le CNES et réalisé par Thalès Alenia Space, dans le cadre d'une coopération avec EUMETSAT. EUMETSAT exploite l'instrument en vol avec le support technique du CNES. La mission IASI a été prévue initialement pour durer 15 ans avec la fabrication de trois modèles de vol (trois instruments identiques destinés à être lancés dans l'espace) : le $1^{\mathrm{er}}$ modèle a été lancé en octobre 2006 à bord de MetOp-A, le second a été lancé en septembre 2012 à bord de MetOp-B, et le lancement du troisième modèle sur MetOp-C est prévu pour 2018.
\end{abstract}

\section{Mission}

IASI permet de sonder l'atmosphère dans le domaine infrarouge sur une couverture mondiale avec une grande précision, et ceci 2 fois par jour ( 9 h30 et $21 \mathrm{~h} 30$ heure locale) : soit 1,2 million de spectres atmosphériques mesurés chaque jour. IASI mesure le rayonnement infrarouge émis en permanence par le système Terre/atmosphère, un exemple de spectre IASI est présenté à la figure 1. Les molécules présentes dans l'atmosphère absorbent ou émettent du rayonnement à des longueurs d'onde très précises, l'intensité du spectre à ces nombres d'onde est caractéristique de la température et de la quantité de molécules présentes dans l'atmosphère.

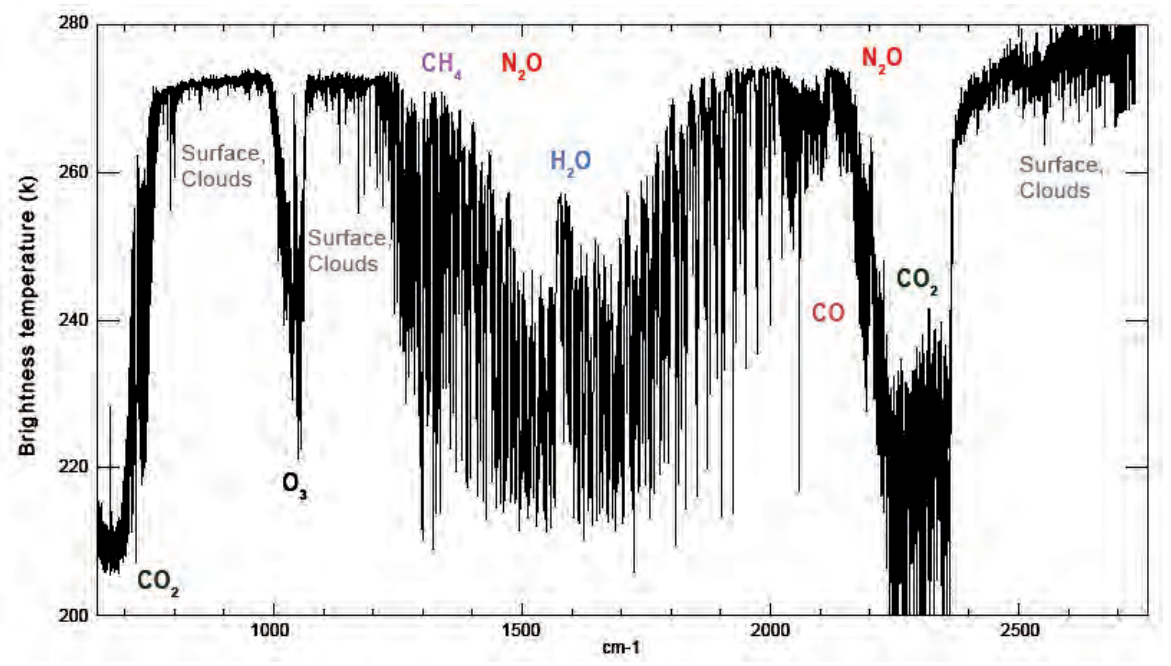

Figure 1. Exemple de spectre IASI, avec les principaux contributeurs aux signatures spectrales.

La mission principale de IASI [1] est de fournir des mesures de profils de température et d'humidité pour la prévision météorologique avec une précision de $1 \mathrm{~K}$ pour la température et de $10 \%$ pour I'humidité avec une résolution verticale de $1 \mathrm{~km}$. Ces données sont utilisées comme état initial dans les modèles numériques de prévision météorologique, et leur précision influence directement la qualité des prévisions. Actuellement la majorité des centres de prévision météorologique du monde (dont Météo France, UK MetOffice ou le centre européen ECMWF) utilisent les données IASI tant dans les modèles globaux que dans les modèles d'échelle fine.

Au-delà de la mission opérationnelle " météo », IASI permet également d'étudier la composition chimique de l'atmosphère : une vingtaine de gaz trace (gaz dont la concentration est inférieure à $1 \%$ du volume de l'atmosphère), dont notamment l'ozone $\left(\mathrm{O}_{3}\right)$, le protoxyde d'azote $\left(\mathrm{N}_{2} \mathrm{O}\right)$ et le monoxyde de carbone (CO) (présenté à la figure 2a), et même l'ammoniac $\left(\mathrm{NH}_{3}\right)$ ou le dioxyde de souffre $\left(\mathrm{SO}_{2}\right.$, lié aux éruptions volcaniques par exemple) sont détectables, voire quantifiables, grâce à la qualité radiométrique et spectrale des mesures IASI.

Enfin les performances en étalonnage absolu de IASI et sa longue durée d'exploitation (plus de 15 ans avec trois missions successives de 5 ans chacune) permettent de constituer des séries temporelles de plusieurs variables climatiques essentielles parmi lesquelles on peut citer : la température, l'humidité, les nuages, les aérosols, les propriétés des surfaces continentales (la température de 


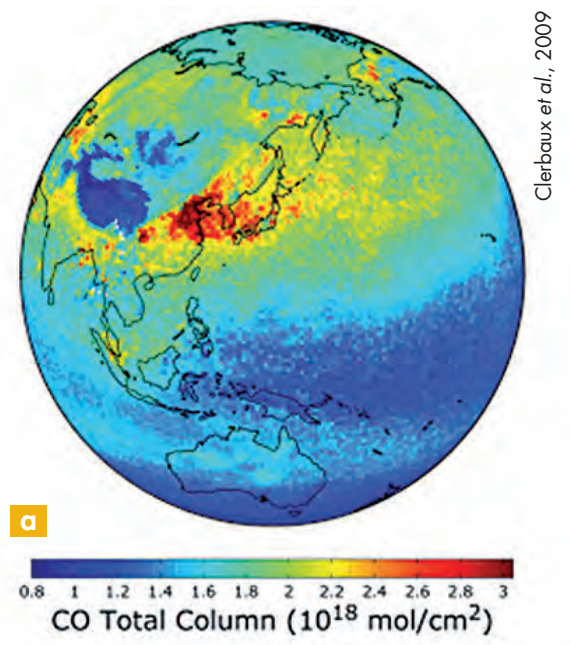

CO Total Column $\left(10^{18} \mathrm{~mol} / \mathrm{cm}^{2}\right)$

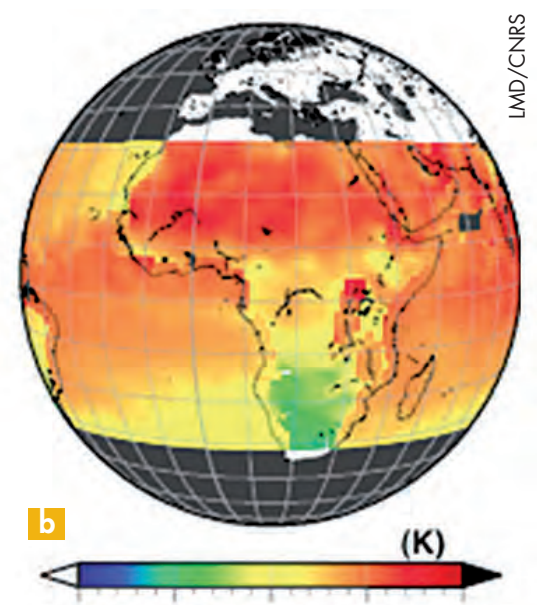

270280290300310

Figure 2. (a) Colonne totale de CO. (b) Température de surface, juillet 2008.

surface comme présentée à la figure $2 b$, ou l'émissivité) et les gaz à effet de serre influencés par les activités humaines comme le dioxyde de carbone $\left(\mathrm{CO}_{2}\right)$ et le méthane $\left(\mathrm{CH}_{4}\right)$. IASI permet le suivi de ces différentes variables climatiques de jour comme de nuit, sur terre et en mer.

\section{De la mission à l'instrument}

La qualité des mesures IASI tient à quelques spécifications clefs au niveau de l'instrument [1]:il mesure le spectre infrarouge émis par le système Terre/atmosphère sur une bande spectrale continue de $645 \mathrm{~cm}^{-1}$ à $2760 \mathrm{~cm}^{-1}$ (i.e., de 3,62 $\mu \mathrm{m}$ à $15,5 \mu \mathrm{m})$, avec un échantillonnage spectral de $0,25 \mathrm{~cm}^{-1}$, une résolution spectrale

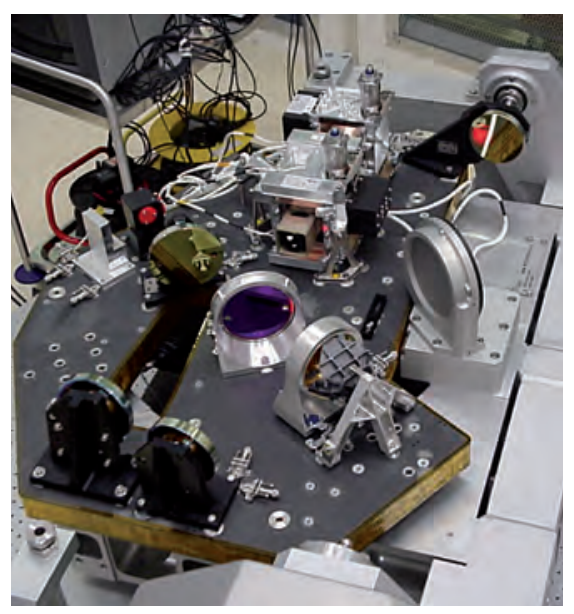

Figure 3. Maquette du cœur interférométrique. de $0,5 \mathrm{~cm}^{-1}$ au niveau utilisateur, avec une stabilité relative de l'étalonnage spectral de $2.10^{-6}$ et une stabilité de la forme de la réponse de l'instrument assurée sur la durée de vie grâce à des corrections au sol basées sur un modèle de l'instrument. Le bruit radiométrique exprimé en NEdT (noise equivalent delta temperature) devant une scène à $280 \mathrm{~K}$ varie en fonction du nombre d'onde de $0,28 \mathrm{Kà} 645 \mathrm{~cm}^{-1}$ et de $0,47 \mathrm{Kà} 2400 \mathrm{~cm}^{-1}$. La précision de l'étalonnage radiométrique est de $0,5 \mathrm{~K}$ à $280 \mathrm{~K}$ dans chaque canal spectral.

\section{Instrument}

L'instrument IASI est un spectro-radiomètre infrarouge basé sur le principe de l'interféromètre de Michelson. Pour tenir les performances radiométriques de la mission, le champ instantané de l'instrument couvre $4(2 \times 2)$ pixels sondeurs de $12 \mathrm{~km}$ de diamètre chacun pour un champ total au sol de l'ordre de $50 \mathrm{~km}^{2}$, ce qui oblige l'interféromètre à travailler hors de l'axe optique. Les pixels sondeurs dans le champ voient une différence de marche dépendant du champ (en cosinus de l'angle du champ) et du fait de l'intégration spatiale due à l'étendue dans le champ des pixels de sondage, l'efficacité de modulation des franges d'interférences diminue à grande différence de marche, ce qui limite les performances spectrales du sondeur. Le point de fonctionnement

\section{Un bond \\ dans le nano positionnement par système piezoélectrique -}

Lancement de la série $\mathbf{q d}$ derotech: nanopositionneur piezo électrique QNP et Piezo contrôleur QLAB

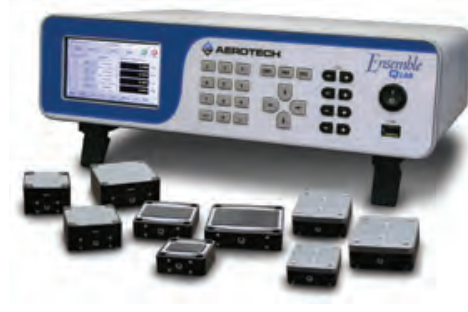

Les tables QNP présentent une raideur hors norme grâce à une fréquence de résonnance très élevée et une résolution subnanométrique. Elles sont donc idéales pour les applications pointues à faible encombrement telles que l'intérferometrie, la microscopie et les alignements d'extrême précision. Le contrôleur associé QLAB dispose d'un écran tactile et peut fonctionner de manière indépendante ou peut être connecté à un $\mathrm{PC}$ via Ethernet, ce qui le rend extrémement flexible dans toutes les situations. Avec des performances sub-nanométriques et un environnement de contrôle et de programmation très convivial, obtenir un positionnement nanométrique n'aura jamais été aussi facile.

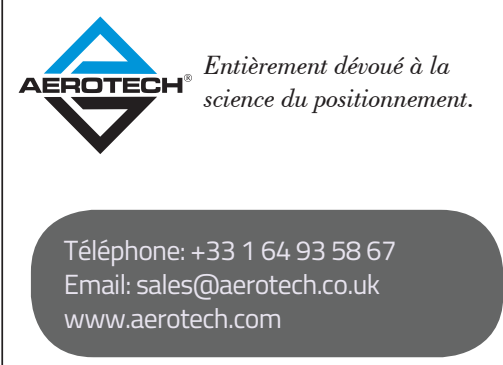

Visitez go.aerotech.com/Q-Series14 pour en savoir plus. 

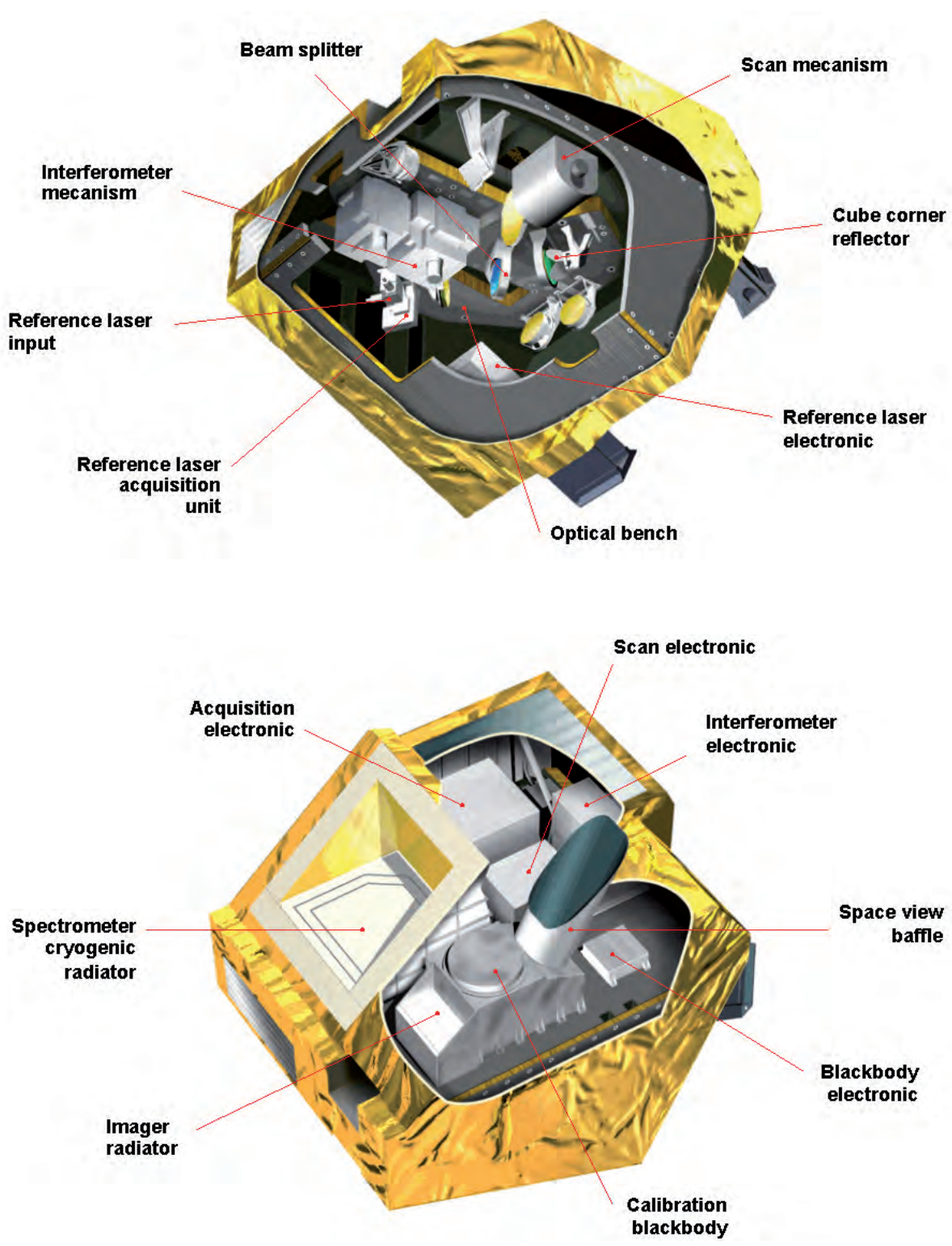

Figure 4. Schémas de l'instrument IASI.

de IASI est un optimum permettant d'atteindre à la fois les performances radiométriques et spectrales.

La mise en œuvre de ce concept instrumental CNES a été confiée à Thalès Alenia Space [2]. La figure 4 illustre la conception de l'instrument. Le miroir de scan, développé par RUAG, comporte 2 axes : il permet de balayer la fauchée de l'instrument (couvrant environ $2000 \mathrm{~km}$ ) sur $90^{\circ}$. Le télescope d'entrée composé de deux miroirs paraboliques assure un filtrage des entrées thermiques et met en forme le faisceau pour entrer dans l'interféromètre. Elément clef du cœur interférométrique, la lame séparatrice est en $\mathrm{ZnSe}$, le traitement séparateur multicouche réalisé par SAGEM-REOSC permet d'obtenir un équilibre des voies transmises et réfléchies de 3,6 $\mu \mathrm{m}$ à $15,5 \mu \mathrm{m}$. Pour limiter les pertes radiométriques à 15,5 $\mu \mathrm{m}$, la lame compensatrice en ZnSe est déportée dans le bras réfléchi de l'interféromètre. Les deux miroirs de l'interféromètre sont des coins de cube en ZERODUR traités or, pesant seulement $170 \mathrm{~g}$ pour un diamètre de $90 \mathrm{~mm}$ (réalisation SAGEM-REOSC). Un des coins de cube est fixe tandis que l'autre est porté par un mécanisme, (réalisé par CSEM), qui assure une translation parfaitement rectiligne de $2 \mathrm{~cm}$ pendant les $152 \mathrm{~ms}$ d'acquisition de

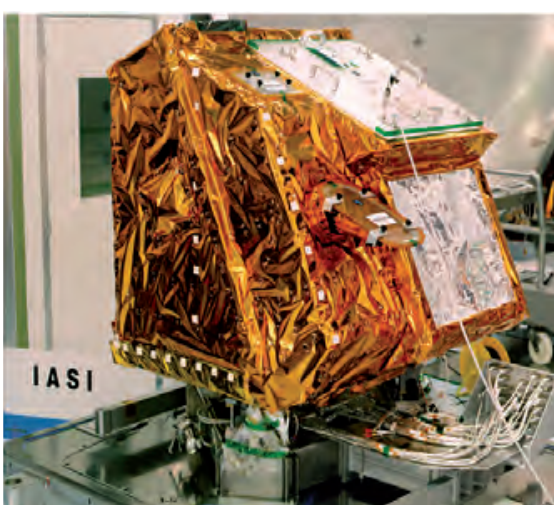

Figure 5. L'instrument IASI en intégration.

l'interférogramme, sur un cycle durant $216 \mathrm{~ms}$ (le temps supplémentaire est utilisé pour inverser le sens de déplacement du coin de cube mobile), soit pas moins de 146 millions d'allers-retours par an.

En sortie de l'interféromètre, le faisceau est repris par un miroir parabolique hors axe pour faire l'image du champ de vue dans la boîte froide. C'est dans cette partie de l'instrument, refroidie passivement, que sont définis le champ et la pupille de l'instrument. Deux lames dichroïques en ZnSe permettent de séparer le faisceau suivant les 3 bandes spectrales et de les focaliser sur les détecteurs monoéléments réalisés par SAT-SAGEM. En bande $1\left(645-1230 \mathrm{~cm}^{-1}\right)$ se trouve un boitier de détection basé sur un détecteur de type photoconducteur à grande longueur d'onde de coupure, en bande $2\left(1150-2050 \mathrm{~cm}^{-1}\right)$ ce sont des photodiodes en $\mathrm{HgCdTe}$ et en bande 3 (1900 - $2760 \mathrm{~cm}^{-1}$ ), ce sont des photodiodes en InSb qui sont utilisées comme puces détectrices. Les détecteurs sont hybridés à un préamplificateur faible bruit travaillant à température cryogénique conçu et fourni par le CNES. L'imagerie de IASI est une imagerie pupillaire : c'est l'image de la pupille d'entrée située au niveau du miroir de scan, qui est faite sur les détecteurs permettant ainsi de dé-contraindre dans une certaine mesure les besoins en uniformité spatiale. Compte tenu de la géométrie des 4 pixels sondeurs, chaque plan focal comprend 4 zones détectrices devant chacune desquelles se trouve une microlentille pour focaliser le signal.

La chaine de détection permet de filtrer la composante ligne de base de 
l'interférogramme optique et d'acquérir le signal à des pas constants en différence de marche. Ce signal d'acquisition est obtenu grâce à un sous-système métrologique qui s'articule autour d'une source laser stabilisée à 1,55 $\mu \mathrm{m}$ (réalisée par la Sodern avec une source fournie par (SO) et d'une chaine de détection détectant les passages à 0 de la modulation du signal métrologique à 1,55 $\mu \mathrm{m}$. L'échantillonnage des 12 signaux « interférogrammes » (4 pixels sondeurs, dans 3 bandes spectrales) à un pas de $775 \mathrm{~nm}$ vérifie les conditions de Shannon ce qui permet de reconstituer les spectres atmosphériques sans repliement par la simple transformée de Fourier inverse de l'interférogramme.

Une dernière prouesse réalisée sur I'instrument IASI concerne le traitement « bord » (i.e., à bord du satellite). En effet, pour des raisons de débit de télémesure et compte tenu de la quantité de données générées par l'instrument, il a été indispensable de passer de l'interférogramme au spectre à bord (permettant de passer de $45 \mathrm{Mbit} / \mathrm{s}$ d'interférogrammes bruts à 1,5 Mbit/s de spectres de niveau 0 ) et d'assurer par voie de conséquence l'étalonnage radiométrique à bord. Ainsi le processeur bord effectue une transformée de Fourier et étalonne les spectres mesurés avec les spectres des visées sur le corps noir interne et l'espace froid (assimilé à un corps noir froid).

Les mesures d'étalonnage radiométrique sont mises à jour à chaque fauchée (toutes les 8 secondes), ce qui permet de s'affranchir des variations thermiques de l'instrument sur l'orbite. L'étalonnage spectral de l'instrument quant à lui s'appuie sur la stabilité et la qualité du laser bord et également sur la mesure physique ellemême et en particulier les raies du $\mathrm{CO}_{2}$ stratosphériques (autour de $2370 \mathrm{~cm}^{-1}$ ) car elles sont visibles dans chaque spectre mesuré et permettent de mettre à jour le modèle de l'instrument implémenté dans le traitement sol. Ce modèle est utilisé en temps réel pour « dé-spatialiser » les mesures: c'est-à-dire en enlever tous les biais instrumentaux et générer des données de niveau $1^{1}$ qui sont utilisées par les centres de météorologie et par les scientifiques.

L'instrument IASI est reconnu aujourd'hui par la communauté internationale des utilisateurs pour ses performances qui lui permettent de remplir pleinement ses missions opérationnelles et scientifiques. En particulier, l'exceptionnelle stabilité spectrale et radiométrique de IASI en a fait l'instrument de référence pour l'inter-étalonnage des instruments d'observation de la Terre dans le cadre de l'effort international du GSICS (qui s'assure de l'inter-étalonnage entre les différents capteurs en vol).

\section{La suite}

Actuellement les 2 instruments IASI sur MetOp-A et MetOp-B sont opérés par EUMETSAT, et la qualité des mesures et les anomalies sont gérées par le centre d'expertise du CNES à Toulouse, centre qui aura également en charge la phase d'étalonnage et validation de l'instrument lors du lancement du $3^{\mathrm{e}}$ modèle de vol. Ce dernier, après 10 ans de stockage au sol, va subir quelques réparations avant d'être lancé en 2018.

À la fin de la mission IASI, la continuité des mesures sera assurée par un nouvel instrument, IASI-NG, qui cherche à faire deux fois mieux que son grand frère en résolution spectrale et en rapport signal à bruit. Il est actuellement en phase de développement et sera lancé en 2021.

Pour plus de renseignements, voir le site internet des missions scientifiques du CNES : http://smsc.cnes.fr/IASI

'Les données $|A S|$ de «niveau 1 » correspondent à des spectres atmosphériques complètement étalonnés et directement utilisables par les scientifiques, contrairement aux données de "niveau 0 » en sortie $d^{\prime}$ 'instrument qui ne sont pas complètement étalonnées et difficilement exploitables.

\section{Références}

[1] Cayla F., «L'interferomètre $\mid A S I$ », La météorologie $8^{e}$ série, $n^{\circ} 32$, p 23-39, février 2001.

[2] Siméoni D. \& al., "Design and development of IASI instrument", Proc. SPIE 5543, Infrared Spaceborne Remote Sensing XII, novembre 2004

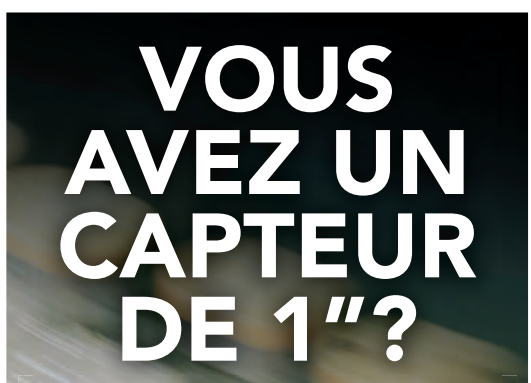

Nous avons les objectifs qu'il vous faut livrables immédiatement!

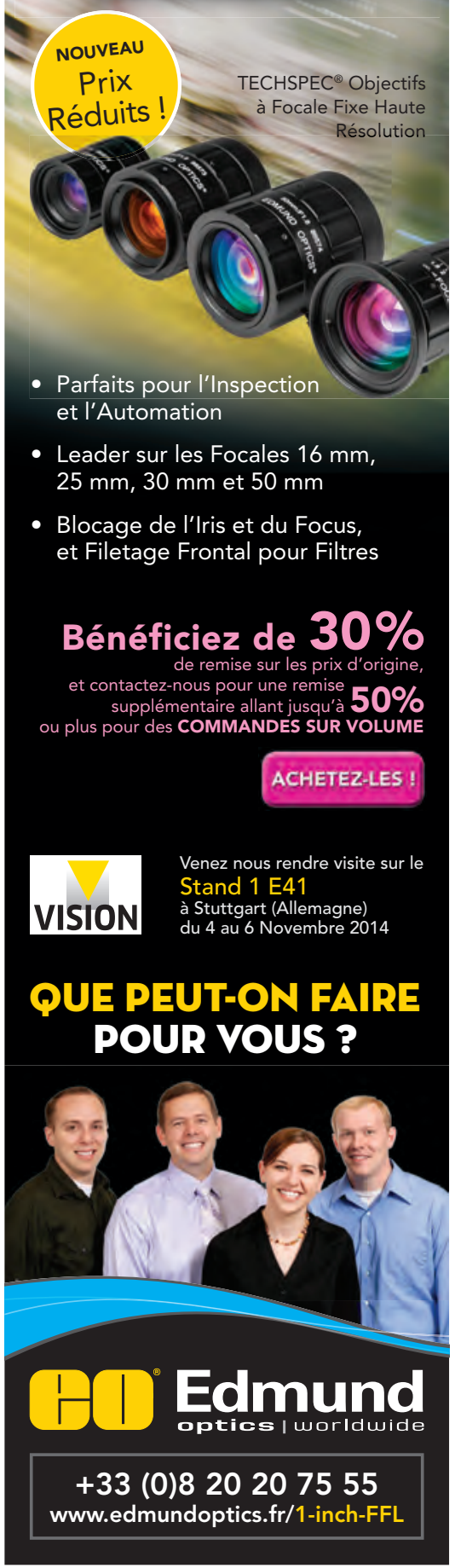

